\title{
Racial Disparities in Cardiovascular Care: A Review of Culprits and Potential Solutions
}

\author{
Quinn Capers IV • Zarina Sharalaya
}

Received: 28 January 2014 / Revised: 25 March 2014 / Accepted: 30 April 2014 / Published online: 23 May 2014

(C) W. Montague Cobb-NMA Health Institute 2014

\begin{abstract}
Cardiovascular disease is the leading cause of death for white and black Americans. Despite the presence of therapies that improve survival and quality of life in cardiac patients, African-Americans continue to have the worst heart disease survival rates of all racial groups. The literature has described racial disparities in cardiovascular care for at least 25 years. We review the current status of racial disparities in four areas of cardiovascular care: automatic implantable cardioverterdefibrillator (AICD) implantations in patients at risk for sudden cardiac death, cardiac resynchronization therapy with defibrillator (CRT-D) implantation in patients with severely depressed left ventricular function and refractory heart failure, reperfusion therapy in patients presenting with acute myocardial infarction, and revascularization in patients with critical limb ischemia. We will discuss potential culprits for these disparities with a particular focus on hospital quality, physician bias, and the relative lack of diversity among cardiovascular physicians in the USA. Finally, we will discuss strategies already in progress that hold promise to reduce or eliminate racial disparities in cardiovascular care.
\end{abstract}

Keywords Racial · Disparities · Black · White - Bias · Cardiovascular · Disparity · Underrepresented · Minority · AICD $\cdot$ Stent $\cdot$ Myocardial infarction $\cdot$ Amputation

\section{Introduction}

Cardiovascular disease is the leading cause of death in both white and black Americans [1]. Racial disparities in

Q. Capers IV $(\bowtie) \cdot$ Z. Sharalaya

Division of Cardiovascular Medicine, The Ohio State University College of Medicine, 370 W. 9th Avenue, Columbus, OH 43210, USA

e-mail: quinn.capers@osumc.edu

Z. Sharalaya

e-mail: Zarina.Sharalaya@osumc.edu cardiovascular care have been documented in the medical literature for over two decades $[2,3]$. Yet, in studies on physician awareness of this issue, physician awareness is reportedly low. A 2005 study reported the results of a survey of attending cardiologists questioned about racial disparities in cardiac care [4]. Only $33 \%$ of the physicians believed that racial disparities exist in health care in general, with only $5 \%$ reporting awareness of such disparities in their own practice. A more contemporary study focused on awareness of racial disparities among internal and emergency medicine residents rotating through a coronary care unit [5]. The residents completed a survey on quality and disparities in cardiovascular care before and after attending a $1 \mathrm{~h}$ seminar on racial disparities in the USA. Prior to the seminar, $35 \%$ of residents believed that racial disparities existed on a national level, and $7 \%$ believed they had personally cared for a patient who experienced a racial disparity in treatment. After the short educational seminar, $85 \%$ of residents acknowledged racial disparities in the US health system, and $32 \%$ agreed that disparities are present on an individual level. Given that nearly 200 publications between 1983 and 2003 documented racial and ethnic disparities in the treatment of acute myocardial infarction [6], the lack of awareness among fully trained heart specialists and trainees documented in these reports is staggering. At the time of preparation of this manuscript, our MEDLINE database search did not reveal more current references on physician awareness of racial disparities in heart care, indicating that this is an area in need of further investigation. Here, we will review racial disparities in the utilization of four therapeutic cardiovascular procedures: automatic implantable cardioverter-defibrillator (AICD) implantation in patients surviving or at risk for sudden cardiac death, cardiac resynchronization therapy with defibrillation (CRT-D) in heart failure patients, reperfusion therapy in patients suffering from acute myocardial infarction, and mechanical revascularization procedures in patients with critical lower extremity ischemia. 
We will discuss potential culprits of these disparities with a particular focus on hospital quality, physician bias, and the relative lack of diversity among cardiovascular physicians in the USA. Finally, we will discuss strategies already in progress that hold promise to reduce or eliminate racial disparities in cardiovascular care.

Automatic implantable cardioverter-defibrillators (AICD) have been shown to prolong life in cardiac arrest survivors [7], and after the publication of major trials that define this survival benefit, the annual number of implants increased dramatically [8]. Groeneveld [9] and colleagues found that although AICD implantation improved long-term survival in the group of cardiac arrest survivors that they examined, there were racial disparities in the use of this technology. Black patients were less likely to receive an implant than their white counterparts with an odds ratio (OR) of 0.58 , a discrepancy that was associated with reduced long-term survival in the black patients. The fact that the study was conducted solely in Medicare recipients makes it less likely that this discrepancy was due to ability to pay. In another study examining cardiovascular care of about 50,000 veterans, it was determined that black patients at Veterans Affairs academic hospitals who were at risk for cardiac arrest were less likely than white veterans at similar medical centers to be treated with prophylactic AICD implantation (OR 0.54) [10]. Disparities in AICD implantation by race have also been demonstrated at hospitals participating in national quality improvement programs. Reviewing outcomes of patients treated at hospitals participating in the American Heart Association "Get With the Guidelines" program, Hernandez and colleagues [11] revealed that of approximately 13,000 heart failure patients with a severely diminished left ventricular ejection fraction of $<30 \%$ - prime candidates for sudden cardiac death and patients for whom AICD implantation is a Class I indication [12] $-28.2 \%$ of black women and $33.4 \%$ of black men received an AICD, compared to $43.6 \%$ of white men. After adjusting for patient characteristics, hospital factors, and study population, the odds ratio for AICD implantation in black women compared to white men was 0.54 . A recent study by Thomas et al. [13] reviewed a possible link between racial differences in preference of AICD therapy by employing a video decision aid. The video was shown to patients who were candidates for AICD implantation, and included patient testimonials, animations, and a multidisciplinary team of nurses and physicians describing the procedure's risks and benefits. Half of the patients in the study were randomized to receive counseling with the video approach, and half were randomized to a control group receiving health care provider counseling without a video. The video decision aid decreased the racial disparity in patient preference for an AICD. However, black patients in the control group were still less likely than white patients to prefer the procedure to medical therapy.
These studies suggest that black patients that have survived or who are at risk for sudden cardiac death are only about half as likely as their white counterparts to be treated with a device that has been demonstrated to prolong life. This disparity does not appear to be impacted by payer mix or veteran status. There is some suggestion that patient preference may play a role, and that this may be remediable through patient education efforts.

Cardiac resynchronization therapy with defibrillation (CRT-D) is another key therapy for patients with advanced heart failure that reduces hospitalizations and mortality in this population [14]. It is notable that even though CRT-D is a newer therapy in cardiovascular medicine, its application follows a similar trend as earlier therapies regarding its underutilization in particular racial groups. In order to study current trends in CRT-D use by race, Eapen and colleagues [14] evaluated 107,096 patients from the National Cardiovascular Data Registry receiving an AICD who were also eligible for CRT-D therapy. While there was a significant increase in CRT-D implantation over the 4-year study among whites, blacks, and Hispanics, the odds ratio for CRT-D implantation was 0.69 for black patients vs. white patients and 0.84 for Hispanic patients vs. white patients. This trend was also seen in a sub-analysis of Medicare patients between white and black patients, essentially negating ability to pay as a contributing factor. A similar study was conducted by Farmer et al [15] to identify potential racial disparities in CRT-D use in patients who receive an AICD, while also evaluating trends in out-of-guideline use of CRT-D among different races. Current criteria for CRT-D eligibility are QRS duration $>120 \mathrm{msec}$, left ventricular ejection fraction $<35 \%$, New York Heart Association Class 3 or 4 congestive heart failure (CHF), and optimal medical management for heart failure [16]. The study included 22,205 patients of white, black, and Hispanic ethnicity who had an ICD implanted and were also eligible for CRTD. After adjusting for multiple confounding variables, results indicated that black and Hispanic patients were less likely to receive CRT-D than white patients with OR of 0.84 and 0.83 , respectively. In conducting a guideline-based analysis on recipients of CRT-D, black and Hispanic patients were more likely to meet clinical guidelines than white patients at an adjusted odds ratio of 1.18 and 1.17 , respectively. Thus, despite black and Hispanic patients meeting eligibility criteria more often, they were still less likely than white patients to received CRT-D therapy.

There are numerous reports documenting racial disparities in the treatment of acute and chronic coronary artery disease. Coronary reperfusion therapies such as thrombolytic therapy and percutaneous coronary intervention (PCI) have dramatically improved survival in acute myocardial infarction patients [17]. Despite this, a study by Manhapra et al [18] showed that black patients suffering from a myocardial infarction who were eligible for thrombolytic therapy were 
significantly less likely than whites to receive it. In a study of 10,469 black patients diagnosed with an ST segment elevation acute myocardial infarction (STEMI) and eligible for thrombolytic reperfusion therapy, $47 \%$ of these patients did not receive any therapy. Fifty-five percent of these black patients were 70 years of age or older, and in this older group twothirds were not treated with reperfusion therapy. In a report by Peterson and colleagues [19], the records of 33,641 male veterans hospitalized in a VA facility for acute myocardial infarction were analyzed to determine utilization rates of major coronary interventions. Overall, black veterans were less likely to receive major coronary procedures than white veterans within 90 days of an acute myocardial infarction. Black veterans were also $33 \%$ less likely to have a diagnostic cardiac catheterization, $42 \%$ less likely to undergo percutaneous coronary intervention, and $54 \%$ less likely to have a coronary bypass surgery. Thus, with regards to coronary reperfusion in acute myocardial infarction, data suggest that blacks are less likely than whites to receive invasive and therapeutic procedures.

Racial disparities in treatment have also been demonstrated among chest pain patients deemed to be low to intermediate risk. Napoli et al. [20] conducted a retrospective observational study of 2,451 patients who presented to the emergency department with low to intermediate risk chest pain to determine if there were racial disparities in stress testing. Patients were also required to have non-dynamic electrocardiograms and non-elevated initial troponin I levels. Thrombolysis in Myocardial Infarction (TIMI) and Diamond \& Forrester (D\&F) scores were calculated for all participants. After controlling for patient insurance, black patients were much less likely to have stress testing than white patients, with odds ratios of 0.68 (TIMI) and 0.67 (D\&F). In a separate statewide study in Michigan, Khambatta et al. [21] performed an analysis of all patients with coronary artery disease (CAD) undergoing percutaneous coronary intervention (PCI). A total of 65,175 patients across 44 different hospitals were included in the study with findings revealing that black patients were less likely than white patients to receive adjunctive therapy with prasugrel (10\% vs. $14.5 \%)$, the adjunctive antiplatelet drug with the highest efficacy, and blacks were also less likely than whites to receive a drug-eluting stent (DES), the current gold standard of coronary stents in the absence of a contraindication. The lower rate of use of DES in black patients was still prevalent after adjusting for socioeconomic status and medical comorbidities. Upon further analysis, the authors concluded that the use of prasugrel and DES was lower in hospitals that treat a higher volume of black patients. The above data suggest that blacks are less likely than whites to receive the standard of care across the spectrum of CAD, from stable, low risk chest pain to life-threatening acute coronary syndromes such a STEMI. As in the case with racial disparities in AICD and CRT-D implantation, at least some reports dismiss payer or veteran's status as a major explanation for these disparities in CAD care.

Critical lower extremity ischemia in patients with peripheral arterial disease (PAD) can reduce the quality of life and eventually lead to loss of limbs. Racial disparities in mechanical revascularization procedures in PAD patients have been widely documented. Guadagnoli and colleagues [22] studied a random sample of 19,236 Medicare patients with lower extremity ischemia, and it was discovered that black patients were two times as likely to undergo above-the-knee or belowthe-knee amputation. White patients were nearly twice as likely as black patients to undergo limb-preserving mechanical revascularization procedures like balloon angioplasty, stent placement, or bypass surgery. A separate study by Eslami et al. [23] examined outcomes of 691,833 patients with lower extremity ischemia at an urban hospital. Two thirds of these patients had surgical or invasive procedures of some type and $33.7 \%$ underwent limb amputation. Further analysis revealed that black patients and those with lower incomes were overrepresented in the amputation group. As a result, black patients were 1.9 times as likely to have an amputation compared with white patients, and patients in a low income group were 1.4 times as likely to have an amputation compared with high income patients. Thus, evidence suggests that among Medicare beneficiaries in urban hospitals, blacks with critical lower extremity ischemia are less likely than their white counterparts to receive therapeutic revascularization procedures and more likely to be treated with limb amputation. This disparity is not completely explained by the patient's ability to pay or level of disease at presentation. Racial disparities in PAD treatment among white and black patients have also been documented outside of the USA: Ahmad and colleagues [24] in a study of 161,523 procedures in England showed that black patients had significantly higher rates of lower limb amputation than white patients (OR 1.45). This disparity was especially striking in the female population, where black women were 2.4 times more likely than white women to have lower limb amputations. Interestingly, the disparities persisted after adjustment for age, sex, and social class (considers income and employment). In a similar study, Hughes et al. [25] studied a population of 240,139 patients from the Nationwide Inpatient Sample (NIS) database who presented with critical limb ischemia. Black patients made up $20 \%$ of the total patient sample, however represented $25 \%$ of the total lower extremity amputations performed. Because of the potential confounding effect of prohibitive comorbidities, the study also included an analysis on the relative number of comorbidities between black and white patients. White patients were significantly more likely to have cardiac and pulmonary comorbidities than black patients. In a separate study on racial disparities in PAD care, Durazzo and colleagues [26] investigated access to care and an institution's capacity to perform revascularization procedures as potential 
underlying explanations. Researchers analyzed 774,399 patients from the NIS Database showing that more black than white patients had an amputation to treat critical PAD at rates of 56.4 and $34.5 \%$, respectively. It was discovered that black race alone increased the odds of having an amputation (OR 1.78 ) independent of socioeconomic status or insurance. Astonishingly, the observed racial disparities in amputations between black and white patients occurred more often at institutions with the greatest resources for performing revascularization (OR 1.8). Researchers also addressed the degree of ischemia upon presentation to the hospital to explore the possibility that a more advanced disease presentation may preclude patients from a revascularization procedure. After accounting for this variable in the regression model, the racial disparities in PAD treatment were still observed. In summary, blacks receive disparate treatment of critical limb ischemia with the use of amputation as a treatment modality more often than whites both in the USA and abroad. At least one study suggests that severity of disease is not an explanation of the unequal treatment, as is often suggested.

\section{Potential Culprits}

Acknowledging and understanding the culprits underlying these racial disparities in cardiovascular care are critical to eliminating these inequities. Though not meant to be exhaustive, we list several possible explanations here:

- Some black patients may decline state of the art treatments or fail to seek out care because they are less informed about symptoms, medications, and treatment related to heart disease than their white counterparts.

- Black patients may be less likely to trust the health care system based on their perception of discrimination and knowledge of historical atrocities like the Tuskegee Experiment of Untreated Syphilis in Negro Males.

- Physicians caring for black patients may be overwhelmed by the volume of patients and the severity of disease at presentation, and underperforming with regards to following evidence-based treatments.

- Unconscious bias with regard to race ("white preference") and frank racism may result in physicians being selective in applying medical and procedural therapies.

- Black patients may be overrepresented at hospitals that underperform with regards to high-quality, evidencebased cardiac care.

- The physician workforce in the USA does not reflect the nation's patient population, potentially exacerbating all of the above and depriving many patients of culturally sensitive, compassionate care from individuals with whom they have something in common and whose recommendations they may be more likely to follow.
Although published studies can be found to make a case for each of these being a potential contributor to racial disparities in cardiac care, we will focus on three: physician bias, underperforming "black hospitals", and a lack of diversity among cardiologists and other physicians in the USA.

\section{Physician Bias as a Potential Culprit}

Schulman et al. [27] conducted a study in which physicians viewed videos of actors of various races and genders playing the part of patients. All "patients" had identical or very similar medical histories and clinical presentations suspicious for heart disease; the only variables were race and gender. Each physician viewed one patient vignette, and they were asked to determine when or if cardiac catheterization was indicated. Black women were least likely of all groups to be referred for cardiac catheterization. This study suggests that physician decision-making can be influenced by patient race and gender. Expanding on this concept was a study by Green and colleagues [28] conducted on a group of 220 internal medicine and emergency medicine residents or physicians in training. The doctors took the "Implicit Association Test" for race, a computer-based psychological test to detect unconscious race preference or bias [29]. The resident physicians also reviewed a clinical vignette of a patient presenting with a clinical history of acute myocardial infarction. Half of the residents were randomly assigned to a vignette with a photo of a white man representing the patient while half had the acute MI patient represented by the photo of a black man. The clinical histories were identical. Participants were to answer three questions: whether the presentation seemed consistent with a cardiac cause, whether or not the patient should receive thrombolytic therapy (drug treatment that dissolves clots in coronary arteries and is known to be life-saving in heart attack patients), and whether the patient was expected to be cooperative with the treatment plan. On the measure of self-reported explicit bias, respondents reported no race preference and anticipated both groups to be equally cooperative. However, results of the implicit bias test indicated that white, Asian, and Hispanic residents believed the black patient would be "less cooperative" with the doctor's recommendations. Based on the clinical description, slightly more residents believed the black patient's symptoms to be secondary to coronary artery disease (CAD). Yet, while $60 \%$ of the physicians indicated that they would offer thrombolytic therapy to the white patient, only $40 \%$ of the physicians indicated that they would offer treatment to the black patient. The strongest predictor of the decision not to treat the black heart attack patient was the resident's implicit "white preference" or unconscious negative racial bias about blacks. Thus, the doctor's implicit racial bias showed a strong association with the decision to provide a life-saving therapy. While computer-based tests cannot 
reproduce actual clinical scenarios, these results are disturbing and highlight the possibility of physician implicit racial bias being a potential culprit of the racial disparities in cardiovascular care under discussion. Further analyzing physician implicit physician bias was a study by Blair and colleagues [30] investigating the relationship between patient's perception of care and physician implicit bias. A group of 2,908 patients was surveyed regarding their perception of the quality of patient-centered care they received while the 134 physicians caring for them completed the Implicit Association Test (IAT). The study showed that $43 \%$ of physicians had moderate to strong "white preference" or implicit bias against blacks. The physicians reported very little explicit racial bias. The analysis showed that the stronger the physician's implicit white preference, the lower their black patients rated the care they received. Black patients rated physicians who had strong white preference approximately six points lower on the quality of interpersonal treatment than physicians who had no implicit bias. Thus, physician implicit white racial preference was associated with the perception of reduced quality of interpersonal interaction and an overall feeling of uneasiness with the physician-patient interaction by black patients. The effects of physician implicit racial bias have also been observed in a pediatric population [31]. In a 2012 study by Sabin and colleagues, a group of 86 pediatricians from a large urban research university were provided with case vignettes of various common pediatric ailments. Each case had an element of clinical uncertainty and physicians were required to submit their input on treatment options, from best practice to adequate or "good enough" care. Physicians were also administered the IAT to measure implicit racial bias. The research showed that physicians with more pro-white bias were more likely to prescribe a full course of pain management to white patients than to black patients. For example, physicians with implicit white racial preference were more likely to prescribe narcotics for postsurgical white patients as opposed to postsurgical black patients. Physicians with low implicit bias were more likely to prescribe five extra days of oxycodone, while physicians with high implicit bias were less likely to do so for postoperative patients. These studies imply that physician implicit racial bias can contribute to racial disparities across adult and pediatric fields of medicine.

\section{Disparate Hospital Quality as a Potential Culprit (A Tale of Two Hospitals)}

Data have emerged that seem to tell a story of two classes of hospitals in this country with respect to cardiovascular care: those that follow established treatment guidelines and post excellent outcomes as a result, and those that for a variety of reasons, among them payer mix, strapped finances, and perhaps provider bias, do not. Bradley and colleagues [32] designed a retrospective study estimating differences in door-to-drug (fibrinolytic therapy) and door-to-balloon (PCI) times in heart attack patients who present to a wide variety of hospitals in the USA. They discovered that black patients had $20 \%$ longer times on average than white patients. This equated to $7 \mathrm{~min}$ longer for door-to-drug time and $19 \mathrm{~min}$ longer for door-to-balloon times, critical quality metrics in the treatment of heart attacks. When the differences between hospitals were accounted for (urban vs. rural locations, teaching status, ownership type, cardiac facilities), the gross difference in door-totherapy times between black and white patients was reduced but remained significant. The delays in treatment were largely accounted for by the hospital to which these patients were admitted as opposed to racial disparities in treatment within the hospital itself.

Similar trends have been demonstrated in timely defibrillation postcardiac arrest of hospitalized patients. Depending on the cause of cardiac arrest, defibrillation within 2 min of a ventricular arrhythmia is associated with significantly lower mortality rates [33]. Each additional minute to defibrillation causes a serious decline in survival rates. Chan and colleagues [34] examined data on 369 hospitals and 6,789 patients suffering in-hospital cardiac arrest due to ventricular fibrillation or pulseless ventricular tachycardia. The time to defibrillation was calculated in these cases. It was discovered that black race is a risk factor for delayed defibrillation. After adjusting for patient and hospital characteristics, delayed defibrillation was associated with a significantly reduced likelihood of return of spontaneous circulation as well as a decreased survival rate. In both studies, racial disparities in the rapid defibrillation of inhospital cardiac arrest victims were due to a larger number of black patients receiving care at hospitals with lower postresuscitation survival rates.

Given that lower limb amputation leads to a permanent disability or severe impairment of mobility (not to mention significant mental anguish and depression, at least initially), lower limb amputation in patients with critical lower extremity arterial disease should be considered only after all possibilities have been exhausted. Several authors have documented that blacks are more likely than white patients with critical lower extremity vascular disease to be treated with amputations. This, too, appears to be significantly influenced by the hospitals at which many blacks receive their care. Regenbogen et al. [35] sought to identify whether racial disparities in amputation rates are due primarily to differential treatment between hospital sites or within the hospital itself. They found that among 86,865 Medicare patients undergoing major vascular procedures, black patients were more than twice as likely to have amputations compared to whites. Compared to whites, black patients were more likely to have major vascular surgery performed by non-specialists $(27 \%$ of surgeries performed on white patients were performed by physicians without advanced training in vascular surgery; for the black patients this 
rate was $41 \%$ ). Along similar lines, black patients were more likely to have surgery in hospitals considered to be "lowvolume" regarding the number of annual vascular surgeries, and were nearly twice as likely to have their procedures at hospitals with high amputation rates. These findings suggest that the problem of disparate care can be solved in part by the following strategies: enforcement of evidence-based procedural volume guidelines at underperforming hospitals; having underfunded, under-resourced hospitals team up with better resourced facilities for proctoring or cross training; or establishing regional centers of excellence for major procedures while enhancing access to such high-volume and highperforming centers and providers for all patients. More concerning because it does not lend itself to a practical remedy is the finding that even after correcting for differences in physician credentials and hospital quality, the disparities were not completely eliminated. When the investigators examined data for patients who were referred to high-volume centers with fellowship-trained vascular surgeons with endovascular skills, overall amputation rates were reduced but still nearly double for black versus white patients.

Higher volumes of surgical procedures by individual physicians and hospitals have been positively correlated with improved outcomes, with a direct correlation between volume and quality. This is true for vascular surgery as well as coronary artery bypass grafting (CABG). Recent data indicate that black patients disproportionately receive their care at lowvolume hospitals. To study this phenomenon in the state of New York, Mukamel and colleagues [36] reviewed 11,296 consecutive CABG procedures in 1996 in New York. They found that black patients were more likely than white patients to be referred to surgeons with the highest risk-adjusted mortality rates in comparison with white patients. A separate study by Epstein et al. [37] found that for nine of ten major surgical procedures black patients were less likely to be operated on by a high-volume surgeon at a high-volume center, the best case scenario reflected in the best outcomes. Compared to whites, blacks were more likely to have surgery performed by a lowvolume surgeon at a low-volume hospital - the worst case scenario in terms of procedural outcomes. While disparate referral patterns and issues of unequal access are operational in these results, they also highlight the fact that blacks are overrepresented at hospitals that are underperforming.

In a separate study by Rangrass and colleagues [38], the effect of hospital quality on racial disparities was examined in patients who had a CABG surgery. The primary quality endpoint was 30-day mortality at each hospital. In looking at the data of 173,925 Medicare patients having a CABG procedure, it was discovered that non-white patients had a $33 \%$ greater mortality rate than white patients (OR 1.33). In classifying hospitals based on the number of minorities treated, hospitals with the greatest proportion of non-white patients had the highest risk-adjusted mortality rates, 3.8 vs. $4.8 \%$ for white versus non-white patients. Hospital quality alone accounted for $54 \%$ of the observed racial disparity between black and white patients. After adjusting for comorbidities, hospital quality, and socioeconomic status, $66 \%$ of the observed disparity between black and white patients was explained.

These data tell "a tale of two hospitals" in this country; high-quality hospitals frequented by the majority population, and underperforming hospitals largely frequented by blacks and other minorities that may be associated with longer doorto-balloon times after an acute MI, delayed defibrillation after cardiac arrest, a relatively high volume of limb amputations in PAD patients, lower numbers of physicians with advanced expertise, and lower cardiovascular procedural volumes. Solutions to improve care for all patients regardless of the hospital location at which they receive their cardiac services include enforcing guideline-driven care and algorithms, implementing procedural volume requirements, and mandating that under-resourced hospitals partner with high-quality, high-volume institutions.

\section{Lack of Diversity in Physician Workforce as a Potential Culprit}

For the over 40 million African-Americans living in the USA [39], cardiovascular disease will be the leading cause of death. Physicians from different racial groups can bring unique outlooks to the bedside and provide enhanced care to a multicultural patient population, which may in turn increase patient satisfaction. Moreover, the authors believe that a racially diverse physician workforce has the potential to reduce racial health care disparities. Publications like those by Blair [30] on implicit racial bias and the recent comprehensive review published by the US Department of Health and Human Services (HHS) [40] provide strong rationale that a lack of diversity in the physician workforce contributes to inferior outcomes in minority populations. The HHS publication compiles data that demonstrate that physicians from backgrounds underrepresented in medicine (URM - usually defined as black, Hispanic, American Indian, native Alaskan, or native Pacific Islander) tend to practice in underserved and disadvantaged areas at rates significantly higher than white physicians. This assertion is supported by a publication from our institution by McDougle at al. [41] reporting that URM physician graduates of The Ohio State University College of Medicine who first attended a special postbaccalaureate program ultimately practice in underserved areas and provide charity care at rates significantly higher than their white, non-postbaccalaureate program cohort at our institution.

Given the perceived benefits of doctor-patient race concordance and culturally sensitive interactions on physicianpatient communication, the fact that fewer than $4 \%$ of all American cardiologists are black or African-American is 
cause for concern [42]. Regarding the immediate pipeline, survey data note that in 2010 out of a total of 2,429 trainees in American Council of Graduate Medical Education (ACGME)-accredited cardiology fellowships, only 105 $(4.3 \%)$ were black [43], indicating that proponents for increasing the ranks of black cardiologists should not expect help from the immediate pipeline anytime soon. Prior to specialty training in cardiology, physicians must first graduate from medical school and complete 3 years of training in internal medicine. In 2010, only $7 \%$ of first-year medical school enrollees were black, a number that has remained relatively stable for years [42]. Indeed, it appears that the foundation for health care disparities begins much earlier in the educational and professional pipeline-in high school. According to the National Center for Education Statistics [44], $80 \%$ of white students entering high school ultimately graduate, compared to approximately $60 \%$ of AfricanAmericans, Hispanics, and American Indians entering high school. Not coincidentally, these latter three demographic groups are the same groups traditionally and currently underrepresented in the medical profession and these same groups lag behind others in most cardiovascular health indicators in the US population.

Taken together, these data indicate that blacks are underrepresented in the medical profession and woefully underrepresented in cardiology, and that there is a severe "leak" in the pipeline between the 9th and 12th grades of high school. While these findings do not conclusively prove that a lack of minority physicians contributes to the racial disparities in cardiovascular care being discussed, it is reasonable to assume so based on several pieces of evidence: (1) minority physicians are more likely to treat underserved and impoverished communities and provide charity care; (2) black patients rate the doctor-patient interaction as less favorable when they interact with physicians displaying unconscious "white preference"; (3) patients initially hesitant about invasive cardiac procedures can change their attitudes when they are educated about the risks and benefits — one must assume such sessions are more productive when the patient's perception of the doctor-patient interaction is favorable, as they are when the doctor does not have unconscious racial bias.

\section{Potential Solutions to Reduce the Racial Disparities in Cardiovascular Disease}

\section{Clinical Practice Guidelines}

The variation in the quality of cardiac care noted at different hospitals suggests several logical solutions. One such solution is to encourage, and ultimately enforce, strict adherence to published treatment guidelines in the field of cardiovascular disease. The American Heart Association sponsors a "Get
With the Guidelines" program in which hospitals voluntarily provide data on their patient outcomes by cardiac diagnosis. In analyzing the efficacy of such guideline programs, Cohen and colleagues [45] recently examined 443 hospitals participating in the "Get With the Guidelines-Coronary Artery Disease Program" (GWTG-CAD) over 5 years. The purpose of the study was to analyze disparities in the use of evidence-based care for patients after experiencing an acute MI, and to observe temporal trends in the provision of "defect-free care" in hospitals treating large numbers of black patients. Based on national guidelines, a post MI patient was considered to have received "defect-free care" if they were on aspirin, beta blockers, and statins at discharge and had received revascularization, smoking cessation counseling, and an ACE inhibitor or angiotensin receptor blocker if their ejection fraction was low. While there was a significant gap in the proportion of white and black MI patients receiving defect-free care in the beginning of the observation period, this gap progressively narrowed and ultimately was eliminated by the second half of the study.

In a similar study, Siegel and colleagues [46] highlight the results of the Expecting Success: Excellence in Cardiac Care, a national collaborative project that combined quality improvement efforts with registry data of self-reported patient demographics. The target population was black and Hispanic patients who were discharged with a diagnosis of acute myocardial infarction (AMI) or heart failure (HF). The study included ten hospitals across the USA with high cardiac case volumes and mostly minority patient populations. The hospitals were required to provide a monthly report of quality data sorted according to race, ethnicity, and language. Quality measures that were instituted for AMI and HF treatment included administration of appropriate medications on admission and at discharge, PCI or thrombolytics within a certain time of arrival for AMI, proper discharge instructions, and health counseling. Results indicated that three hospitals had significant racial disparities in AMI and HF management present in the first 6 months of the study, but absent by the conclusion of the study. One institution identified that a small group of community physicians' adherence to clinical guidelines was suboptimal, resulting in racial disparities in treatment. Another institution discovered a large disparity in readmission rates among certain minority groups. In standardizing a self-reported demographic data collection system and linking this to outcomes, the study identified a feasible way for hospitals to track and optimize outcomes across all racial groups.

These studies provide heartening evidence that disparity gaps can be closed in a short period of time. It is foreseeable that equality in cardiovascular care is achievable if hospitals participate in such quality improvement and surveillance programs. We believe that participation in such programs should be mandatory and tied to hospital accreditation and third-party 
reimbursements, and furthermore we propose that the formula for the annual US News and World Report "Best Hospitals" rankings should reward hospitals that participate in such programs.

\section{Diversifying the Physician Workforce}

The US Department of Health and Human Services conducted a review of 55 studies to determine the effects of diversifying the health care workforce [40]. Data indicate that URM physicians serve minority and underserved populations more often, and that when minority patients receive care from physicians of their own race they perceive that the quality of care is higher and, indeed, several markers of quality of care bare this perception out, especially in fields of primary care and mental health. The paper concludes that increasing the diversity of health care professionals would increase access to care for underserved populations. An extensive search of the literature failed to reveal more recent references providing evidence for the positive effects of diversity on health care delivery. Though few doubt that this is the case and numerous anecdotes support this assertion, a dearth of published research reports exists. We join others in calling for more scholarly work specifically addressing the questions "Does diversity in the physician workforce improve health care outcomes?", and "Exactly how does diversity do this?"

It is clear that any programmatic approach to increasing the number of African-American cardiologists will not only need to actively recruit internal medicine residents into cardiology, medical students into internal medicine, college students into medical school, and high school students into college, but will need to spark excitement for science and math in the early years of elementary and junior high school. Various programs by the federal government, colleges and universities, and private foundations have been working for years to successfully enhance the minority pipeline into medicine and health care careers. Along with many other institutions and entities, The Ohio State University (OSU) and its College of Medicine have taken several strides toward diversifying the pipeline of future physicians and potential cardiologists. The University's Young Scholars Program is an initiative to increase the numbers of talented, underrepresented minority students entering college. All candidates are 6th graders from low income families and are in the first generation of his or her family to graduate from a 4-year university of college. Those who successfully complete program requirements throughout middle and high school are guaranteed admission to OSU with a financial aid package based on their individual need. The primary author has worked with community organizations to hold several 1-day "Heart School" programs for underrepresented minority middle school and high school students to expose them to basic anatomy and physiology of the cardiovascular system via dissections and small-group teaching. The Ohio State University College of Medicine also hosts an annual "MD Camp"; a 3-week day camp for underrepresented minority or socioeconomically disadvantaged high school students to interact with medical student mentors, perform research, take classes, and shadow physicians. This focus on the early pipeline is critical, and many institutions participate in programs similar to those described above. Nationwide, efforts such as these need to be maintained, strengthened, studied, and the successful efforts magnified and duplicated. A recent report [47] describes The Ohio State University's successful efforts to diversify its cardiology fellowship training program. Important strategic acts were the following: (1) recruiting, (2) making diversity a top priority when ranking physician candidates for the National Residency Match Program, and (3) incorporating lectures on health care disparities into our cardiology Grand Rounds series. An increased presence of URM students and physicians at The Ohio State University has been achieved in a short period of time, owing to the senior leadership of the institution committing to the idea that diversity is a critical component of institutional excellence. Many other academic institutions have long had commitments to diversity and inclusion and to reducing racial health care disparities. We must accelerate our investments in time and resources in the effort of encouraging URM students to excel in middle and high school, attend college, and enter medical school and cardiovascular medicine fields.

\section{Summary and Conclusions}

Racial disparities in cardiovascular care have been documented for decades, yet they still exist, and awareness of this problem among physicians, even cardiologists, has been low in the face of numerous studies with remarkably similar descriptions of the problem. Potential culprits include physician implicit racial bias, the overrepresentation of black patients at underperforming hospitals, and a paucity of underrepresented minorities in medicine, especially practicing cardiology and related fields, such as cardiac surgery and vascular surgery. We agree with the American College of Cardiology [48] and other entities that elimination of this complex problem will require a multifaceted approach, and many entities, hospitals, academic institutions, and the government have taken action to attempt to reduce these racial disparities. Efforts include stimulating minority youth to engage in science and technological classes at a young age, educating patients on cardiac risk and treatments, educating and "deprogramming" physicians and health care providers to combat implicit negative racial biases, rewarding hospitals for participating in national quality control initiatives that reduce racial disparities, and recruiting minority physicians and medical students into 
cardiovascular fields. These efforts are already underway, but must succeed and proliferate rapidly if we are to finally eliminate this embarrassment to the US health care system.

Conflict of Interest Quinn Capers, IV and Zarina Sharalaya both declare that they have no conflict of interest.

Informed Consent No animal or human studies were carried out by the authors for this article.

\section{References}

1. National Vital Statistics. Centers for Disease Control. 2010.

2. Wenneker MB, Epstein AM. Racial inequalities in the use of procedures for patients with ischemic heart disease in Massachusetts. JAMA. 1989;261:253-7.

3. Ayanian JZ, Udvarhelyi IS, Gatsonis CA, Pashos CL, Epstein AM. Racial differences in the use of revascularization procedures after coronary angiography. JAMA. 1993;269(20):2642-6.

4. Lurie N, Fremont A, Jain AK, et al. Racial and ethnic disparities in care - the perspectives of cardiologists. Circulation. 2005;111: 1264-9.

5. Greysen SR, Siegel B, Sears V, et al. Residents' awareness of racial and ethnic disparities in cardiovascular care. J Grad Med Educ. 2011;3(3):417-20.

6. Geiger HJ. Racial and ethnic disparities in diagnosis and treatment: a review of the evidence and a consideration of causes. In: Smedley B, Stith A, Nelson A, editors. Unequal treatment: confronting racial and ethnic disparities in health care. Washington, DC: National Academies Press; 2003. p. 417-54.

7. Kuck KH, Cappato R, Siebels J, et al. Randomized comparison of anti-arrhythmic drug therapy with implantable defibrillators in patients resuscitated from cardiac arrest: the Cardiac Arrest Study Hamburg. Circulation. 2000;102:748-54.

8. Higgins SL. Impact of the multicenter automatic defibrillator implantation trial on implantable cardiac defibrillator indication trends. Am J Cardiol. 1999;83:79D-82D.

9. Groeneveld PW, Heidenreich PA, Garger AM. Racial disparity in cardiac procedures and mortality among long-term survivors of cardiac arrest. Circulation. 2003;108:286-91.

10. Groenenveld PW, Kruse GB, Chen Z, et al. Variation in cardiac procedure use and racial disparity among Veterans Affairs Hospitals. Am Heart J. 2007;153:320-7.

11. Hernandez AF, Fonarow GC, Liang L, et al. Sex and racial differences in the use of implantable cardioverter-defibrillators among patients hospitalized with heart failure. JAMA. 2007;298(13):1525-32.

12. Epstein AE, DiMarco JP, Ellenbogen KA, et al. ACC/AHA/HRS 2008 Guidelines for device-based therapy of cardiac rhythm abnormalities: a report of the American College of Cardiology/American Heart Association Task Force on Practice Guidelines (writing committee to revise the ACC/AHA/NASPE 2002 guideline update for implantation of cardiac pacemakers and antiarrhythmia devices): developed in collaboration with the American Association for Thoracic Surgery and Society of Thoracic Surgeons. Circulation. 2008;117(21):e350-408.

13. Thomas KL, Zimmer LO, Dai D, et al. Educational videos to reduce racial disparities in ICD therapy Via Innovative Designs (VIVID): a randomized clinical trial. Am Heart J. 2013;166(1):157-63.
14. Eapen ZJ, Al-Khatib S, Lopes RD, et al. Are racial/ethnic gaps in the use of cardiac resynchronization therapy narrowing? JACC. 2012;60(16):1577-8.

15. Farmer SA, Kirkpatrick JN, Heidenreich PA, et al. Ethnic and racial disparities in cardiac resynchronization therapy. Heart Rhythm. 2009;6(3):325-31.

16. Hunt SA. ACC/AHA 2005 guideline update for the diagnosis and management of chronic heart failure in the adult: a report of the American College of Cardiology/American Heart Association Task Force on Practice Guidelines (Writing Committee to update the 2001 Guidelines for the Evaluation and Management of Heart Failure). JACC. 2005;46:e1-e82.

17. McGovern PG, Pankow JS, Shahar E, et al. Recent trends in acute coronary heart disease - mortality, morbidity, medical care, and risk factors: the Minnesota Heart Survey Investigators. N Engl J Med. 1996;334:884-90.

18. Manhapra A, Canto JG, Barron HV, et al. Underutilization of reperfusion therapy in eligible African Americans with acute myocardial infarction: role of presentation and evaluation characteristics. Am Heart J. 2001;142:604-10.

19. Peterson ED, Wright SM, Daley J, et al. Racial variation in cardiac procedure use and survival following acute myocardial infarction in the Department of Veterans Affairs. JAMA. 1994;271(15):1175-80.

20. Napoli AM, Choo EK, Dai J, et al. Racial disparities in stress test utilization in an emergency department chest pain unit. Crit Pathw Cardiol. 2013;12(1):9-13.

21. Khambatta S, Seth M, Rosman HS, et al. The association between patient race, treatment, and outcomes of patients undergoing contemporary percutaneous coronary intervention: insights from the Blue Cross Blue Shield of Michigan Cardiovascular Consortium (BMC2). Am Heart J. 2013;165(6):893-901.

22. Guadagnoli E, Ayanian JZ, Gibbons G, et al. The influence of race on the use of surgical procedures for treatment of peripheral vascular disease of the lower extremities. Arch Surg. 1995;130(4):381-6.

23. Eslami MH, Zayaruzny M, Fitzgerald GA. The adverse effects of race, insurance status, and low income on the rate of amputation in patients presenting with lower extremity ischemia. J Vasc Surg. 2007;45:55-9.

24. Ahmad N, Thomas GN, Chan C, et al. Ethnic differences in lower limb revascularization and amputation rates. Implications for the aetiopathology of atherosclerosis? Atherosclerosis. 2014;233:503-7.

25. Hughes K, Seetahal S, Oyetunji T, et al. Racial/ethnic disparities in amputation and revascularization: a nationwide inpatient sample study. Vasc Endovasc Surg. 2014;48(1):34-7.

26. Durazzo TS, Frencher S, Gusberg R. Influence of race on the management of lower extremity ischemia: revascularization vs amputation. JAMA Surg. 2013;148(7):617-23.

27. Schulman K, Berlin J, Harless W, et al. The effect of race and sex on physicians' recommendations for cardiac catheterization. N Engl J Med. 2003;340:618-26.

28. Green AR, Carney DR, Pallin DJ, et al. Implicit bias among physicians and its prediction of thrombolysis decisions for black and white patients. J Gen Int Med. 2007;22:1231-8.

29. Nosek BA, Greenwald AG, Banaji MR. The Implicit Association Test at age 7: a methodological and conceptual review. In: Bargh JA, editor. Automatic processes in social thinking and behavior. London: Psychology; 2007.

30. Blair IV, Steiner JF, Fairclough DL, et al. Clinicians' implicit ethnic/ racial bias and perceptions of care among black and Latino patients. Ann Fam Med. 2013;11(1):43-52.

31. Sabin JA, Greenwald AG. The influence of implicit bias on treatment recommendations for 4 common pediatric conditions: pain, urinary tract infections, attention deficit hyperactivity disorder, and asthma. Am J Public Health. 2012;102(5):988-95. 
32. Bradley EH, Herrin J, Wang Y, et al. Racial and ethnic differences in time to acute reperfusion therapy for patients hospitalized with myocardial infarction. JAMA. 2004;292:1563-72.

33. Chan PS, Krumholz HM, Nichol G, et al. Delayed time to defibrillation after in-hospital cardiac arrest. N Engl J Med. 2008;358(1):9-17.

34. Chan PS, Nichol G, Krumholz HM, et al. Racial differences in survival after in-hospital cardiac arrest. JAMA. 2009;302(11): 1195-201.

35. Regenbogen SE, Gawande AA, Lipsitz SR, et al. Do differences in hospital and surgeon quality explain racial disparities in lowerextremity vascular amputations? Ann Surg. 2009;250:424-31.

36. Mukamel DB, Murthy AS, Weimer DL. Racial differences in access to high-quality cardiac surgeons. Am J Public Health. 2000;90(11): 1774-7.

37. Epstein AJ, Gray BH, Schlesinger M. Racial and ethnic differences in the use of high-volume hospitals and surgeons. Arch Surg. 2010;145(2):179-86.

38. Rangrass G, Ghaferi AA, Dimick JB. Explaining racial disparities in outcomes after cardiac surgery: the role of hospital quality. JAMA Surg. 2014;149(3):223-7.

39. United States Census Bureau: state and county quick facts. 2011.

40. U.S. Department of Health and Human Services. The rationale for diversity in the health professions: a review of the evidence. 2006. http://bhpr.hrsa.gov/healthworkforce/reports/diversityreview evidence.pdf. (accessed 22 March 2014).
41. McDougle L, Way DP, Rucker YL. Survey of care for the underserved: a control group study of practicing physicians who were graduates of The Ohio State University College of Medicine Premedical Postbaccalaureate Training Program. Acad Med. 2010;85:36-40.

42. Association of American Colleges, diversity in the physician workforce, facts and figures tables. 2010.

43. Brotherton SE, Etzel SI. Graduated medical education, 2009-2010. JAMA. 2010;304:1255-70.

44. National Center for Education Statistics. Common core of data, 20062007.

45. Cohen MG, Fonarow GC, Peterson ED, et al. Racial and ethnic differences in the treatment of acute myocardial infarction: findings from the Get with the Guidelines-Coronary Artery Disease program. Circulation. 2010;121:2294-301.

46. Siegel B, Sears V, Bretsch JK, et al. A quality improvement framework for equity in cardiovascular care: results of a national collaborative. J Health Care Qual. 2012;34(2):32.

47. Auseon A, Kolibash AJ, Capers IV Q. Successful efforts to increase diversity in a cardiology fellowship training program. J Grad Med Ed. 2013;5(3):481-5.

48. Yancy CW, Wang TY, Ventura HO, Piña IL, Vijayaraghavan K, Ferdinand KC, et al. The coalition to reduce racial and ethnic disparities in cardiovascular disease outcomes (credo): why credo matters to cardiologists. J Am Coll Cardiol. 2011;57(3):245-52. doi:10.1016/ j.jacc.2010.09.027. 